\title{
Support and Positioning Mechanism of a Detection Robot inside a Spherical Tank
}

\author{
Chunlei Tu ${ }^{1,2^{*}} \mathbb{0}$, Shanshan Jin ${ }^{1}$, Kai Zheng ${ }^{2}$, Xingsong Wang ${ }^{1}$ and Sichong Sun ${ }^{3}$
}

\begin{abstract}
Large pressure equipment needs to be tested regularly to ensure safe operation; wall-climbing robots can carry the necessary tools to inspect spherical tanks, such as cameras and non-destructive testing equipment. However, a wall-climbing robot inside a spherical tank cannot be accurately positioned owing to the particularity of the spherical tank structure. This paper proposes a passive support and positioning mechanism fixed in a spherical tank to improve the adsorption capacity and positioning accuracy of the inspection robot. The main body of the mechanism was designed as a truss composed of carbon fiber telescopic rods and can work in spherical tanks with diameters of 4.6-15.7 $\mathrm{m}$. The structural strength, stiffness, and stability of the mechanism are analyzed via force and deformation simulations. By constructing a mathematical model of the support and positioning mechanism, the influence of structural deformation on the supporting capacity is analyzed and calculated. The robot positioning method based on the support and positioning mechanism can effectively locate the robot inside a spherical tank. Experiments verified the support performance and robot positioning accuracy of the mechanism. This research proposes an auxiliary support and positioning mechanism for a detection robot inside a spherical tank, which can effectively improve the positioning accuracy of the robot and meet the robotic inspection requirements.
\end{abstract}

Keywords: Support mechanism, Inspection of the spherical tank, Ansys simulation, Robot positioning

\section{Introduction}

Spherical tanks are sealed containers for storing liquids or gases. To ensure their safety, regular maintenance is required. At present, regular maintenance usually utilizes the scaffolding method inside the spherical tank. Some research institutions have developed dedicated manned workbenches for the internal maintenance of spherical tanks. However, these methods require manual detection and maintenance, which are inefficient and safe. Therefore, the wall-climbing robot has become a research hotspot in the field of automatic inspection of spherical tanks.

The adsorption capacity is the basic ability of wallclimbing robots. At present, a variety of adsorption methods have been applied to wall-climbing robots,

\footnotetext{
*Correspondence: tuchunlei1985@163.com

${ }^{1}$ College of Mechanical Engineering, Southeast University,

Nanjing 211189, China

Full list of author information is available at the end of the article
}

mainly including negative pressure adsorption [1-6], permanent magnet adsorption [7-15], thrust adsorption $[16,17]$, and bionic adsorption $[18,19]$.

Wall-climbing robots based on negative pressure adsorption are usually large and structurally complex, impeding adaptation to complex environments. Bionic wall-climbing robots imitate insects and reptiles to achieve wall-climbing but cannot currently meet the requirements of engineering applications. The adsorption capacity of the thrust adsorption robots is not affected by the conditions of the wall surface but the existing thrust adsorption robots usually use propellers or fans, which are large and consume significant energy. Magnetic adsorption robots use permanent magnets or magnetic wheels to climb on a metal wall; these have the advantages of strong adsorption, simple structure, and energy-efficiency.

The appropriate adsorption method should be selected according to the working environment. For the inspection of large metal equipment, such as spherical tanks, 
the permanent magnet adsorption robots can adsorb stably but some influencing factors, such as rusts and welds, affect the adsorption capacity and the inspection robot for spherical tanks requires a more flexible and stable structure.

There are many forms of permanent magnet wallclimbing robots, such as magnetic-wheels climbing robots [20-23], crawler climbing robots [24-27], and omnidirectional-wheels climbing robots [28-31]. Wallclimbing robots by permanent magnet adsorption can be effectively used in bridges and ships for inspection operations.

We have designed a variety of wall-climbing robots in Refs. [32-35], which can effectively climb on the surface of spherical tanks. Common positioning methods, such as GPS, gyroscope, and machine vision, have poor positioning accuracy for the robot owing to the structural particularity of the spherical tanks. This paper proposes a spherical coordinate passive support mechanism fixed to a spherical tank. The mechanism is connected to the wall-climbing robot and provides thrust to the robot via a pre-tightening force. It does not need to consume electric energy and the thrust is not affected by rust or weld height. Thus, the adsorption capacity of the robot was enhanced. Furthermore, since the support mechanism is driven by the robot, there is no need for complicated control to ensure the mechanism follows the robot's movement. Moreover, the position of the robot can be measured by detecting the attitude of the support mechanism.

The remainder of this paper is organized as follows. In Section 2, the structure of the support mechanism and static analysis are presented. In Section 3, the mathematical models of the support mechanism are proposed, with force analysis. In Section 4, the robot positioning scheme based on the mechanism is presented. Experiments on the support and positioning mechanism are presented in Section 5. Finally, conclusions and future work are discussed in Section 6.

\section{Design of the Support Mechanism}

\subsection{Overall Structural Plan}

Figure 1 describes how the support mechanism works inside a spherical tank. The column of the support mechanism is fixed at the spherical tank and the end of the cantilever is elastically connected with the wall-climbing robot and has a rotational degree of freedom.

The column and cantilever are connected via two rotating pairs and the intersection point of the axes of the two rotating pairs is located at the center of the spherical tank. The support mechanism is purely passive. The cantilever is driven by the wall-climbing robot and provides continuous thrust to the robot.

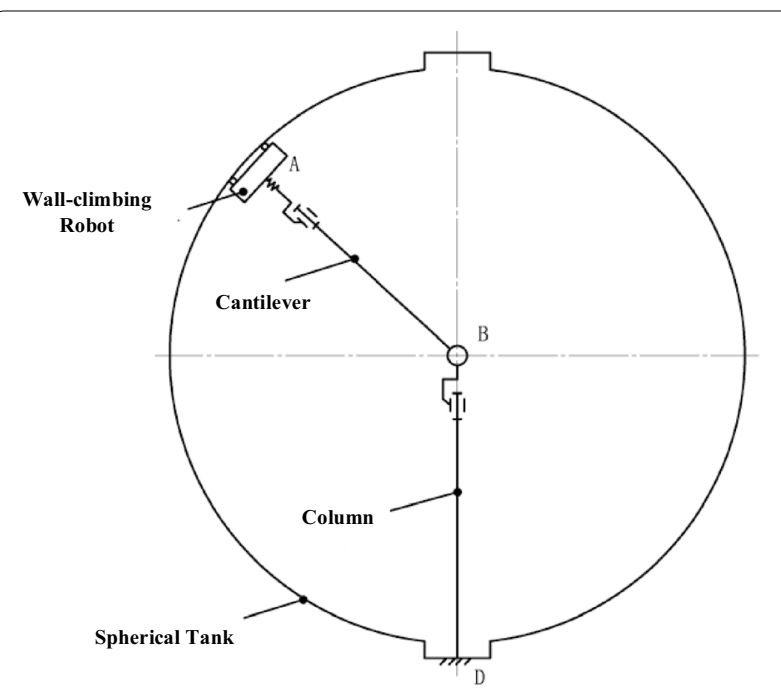

Figure 1 Working principle of the support mechanism

The support mechanism designed in this study can provide $450 \mathrm{~N}$ thrust, the cantilever mass does not exceed $8 \mathrm{~kg}$, and the total weight does not exceed $25 \mathrm{~kg}$. It can adapt to a spherical tank with a volume of $50-2000 \mathrm{~m}^{3}$ (corresponding to a diameter of 4.6-15.7 m).

\subsection{Structural Design}

The overall structural model of the support mechanism is shown in Figure 2. It mainly comprises a cantilever, a column, an elastic pressing mechanism, a supporting base, and a two-degree-of-freedom rotating platform.

The elastic pressing mechanism is at the end of the cantilever and is connected to the climbing wall robot. Its main body is a spring, which can adapt to the slight deformation of the support mechanism during the working process. Simultaneously, adjusting the compression of the spring can change the thrust value. It has a rotation pair at the end so that the robot can adjust its attitude arbitrarily, without the cantilever rotating.

The supporting base is composed of aluminum alloy profiles. As shown in Figure 3, it is bolted to a series of threaded holes in the manhole flange of the spherical tank so that the entire mechanism is fixed to the spherical tank.

The two-degree-of-freedom rotating platform connects the column and cantilever. As shown in Figure 4, it has two rotating degrees of freedom. Two laser rangefinders are installed on the platform to measure the length of the cantilever and column and two angle sensors are used to detect the rotation angles. According to the value of these sensors, the support mechanism can be installed in the correct position quickly and accurately. The sensors also provide a basis for robot localization. 


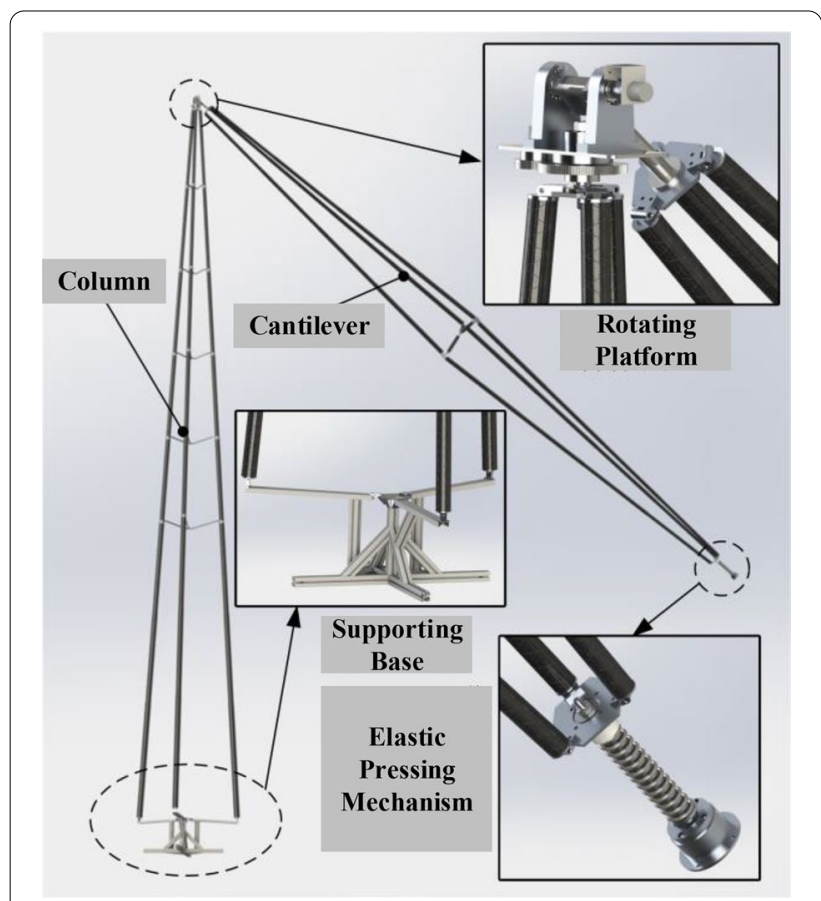

Figure 2 Model of the support mechanism

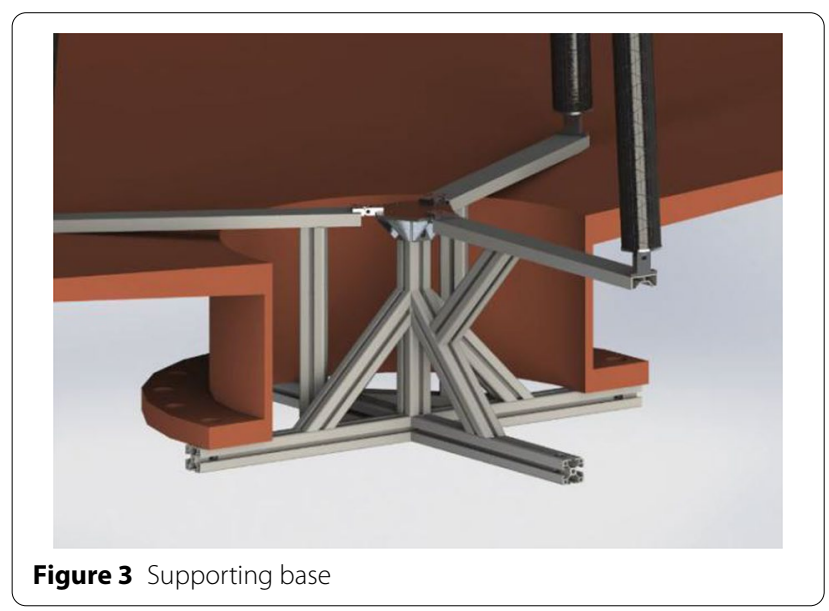

The cantilever and column of the support mechanism are retractable trusses. The main body is composed of carbon fiber telescopic rods and aluminum alloy crossbars. The telescopic rods are connected by an expansion connection mechanism. Through relative rotation between the rods, the telescopic rods are locked and released. When folding, the mechanism can be transported into the tank through manholes. After expansion, the support mechanism can adapt to a spherical tank with a volume of $50-2000 \mathrm{~m}^{3}$, according to the different expansion sizes.

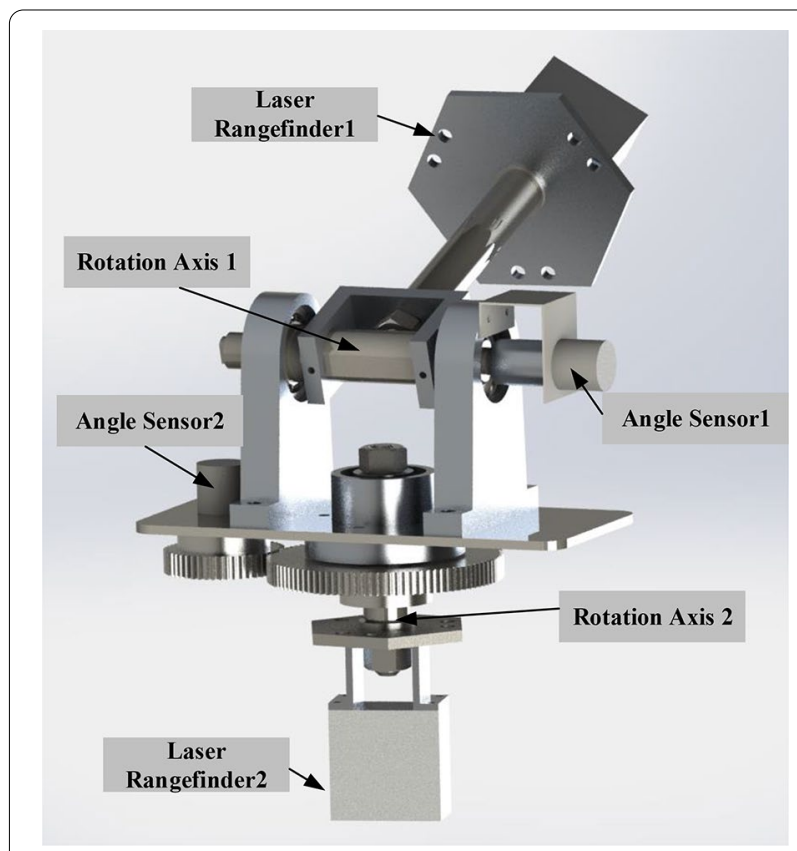

Figure 4 Two-degree-of-freedom rotating platform

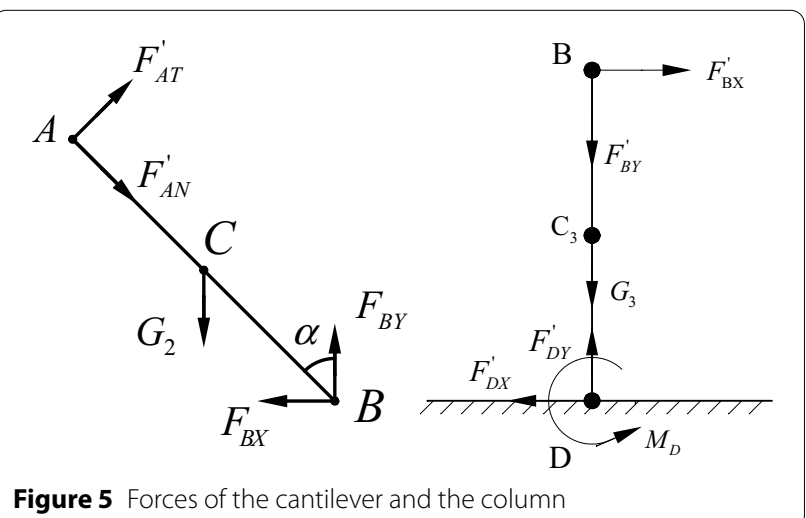

\subsection{Statics Simulation}

The mechanical properties of the mechanism, such as rigidity, are required to be great. To perform a static analysis of the mechanism, a force analysis is first conducted. As shown in Figure 5, $\mathrm{AB}$ and $\mathrm{BD}$ represent the cantilever and column, respectively, and $\mathrm{C}$ and $\mathrm{C}_{3}$ are their barycenters. $\alpha$ is the angle between the cantilever and the vertical direction. The force balance equation of the cantilever and column can be written as:

$$
\left\{\begin{array}{l}
F_{A T}^{\prime} \cos \alpha+F_{A N}^{\prime} \sin \alpha=F_{B X}, \\
F_{A T}^{\prime} \sin \alpha+F_{B Y}=G_{2}+F_{A N}^{\prime} \cos \alpha, \\
M_{B}=G_{2} L_{B C} \sin \alpha-F_{A T}^{\prime} L_{A B}=0,
\end{array}\right.
$$




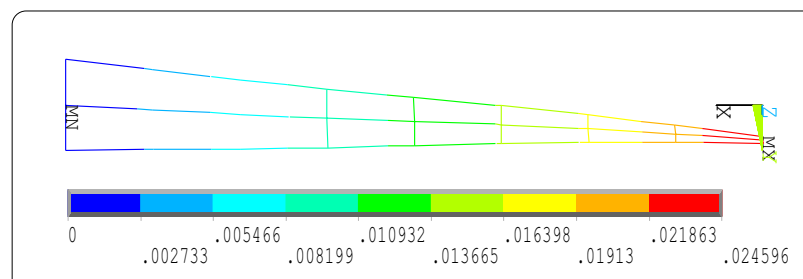

Figure 6 Deformation of the column (mm)

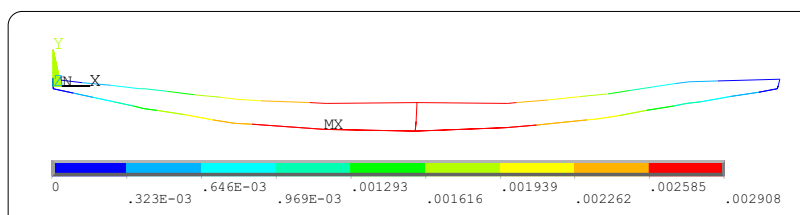

Figure 7 Deformation of the cantilever ( $\mathrm{mm}$ )

\begin{tabular}{|c|c|c|c|c|}
\hline Component & $\begin{array}{l}\text { Maximum } \\
\text { axial } \\
\text { deformation } \\
(\mathrm{mm})\end{array}$ & $\begin{array}{l}\text { Maximum } \\
\text { tangential } \\
\text { deformation } \\
(\mathrm{mm})\end{array}$ & $\begin{array}{l}\text { Maximum } \\
\text { axial } \\
\text { stress } \\
(\mathrm{MPa})\end{array}$ & $\begin{array}{l}\text { Maximum } \\
\text { shear } \\
\text { stress } \\
(\mathrm{MPa})\end{array}$ \\
\hline Column & 0.0503 & 24.59 & 41.17 & 26.75 \\
\hline Cantilever & 0.0752 & 2.908 & 4.05 & 3.48 \\
\hline
\end{tabular}

$$
\left\{\begin{array}{l}
\sum F_{X}=F_{B X}^{\prime}-F_{D X}=0, \\
\sum F_{Y}=F_{D Y}-F_{B Y}^{\prime}-G_{3}=0, \\
\sum M=M_{D}-F_{B X}^{\prime} L_{B D}=0,
\end{array}\right.
$$

where $G_{2}$ and $G_{3}$ are the gravity of the cantilever and column, respectively, and according to the design index, $G_{2} \leq 80 \mathrm{~N}$.

$M_{D}$ is the torque on the terminal position. $L_{B C}, L_{A B}$, and $L_{B D}$ indicate the length of the corresponding lines, and when working inside a spherical tank with a diameter of $15.7 \mathrm{~m}, L_{A B}=L_{B D}=7.85 \mathrm{~m}$.

According to the design index, the support mechanism needs to provide $450 \mathrm{~N}$ thrust; thus, $F_{A N}^{\prime}=450 \mathrm{~N}$, which can be substituted into Eq. (1) and Eq. (2). The calculation result indicates that the bending moments of the column and cantilever are largest when the cantilever is horizontal $\left(\alpha=90^{\circ}\right)$. This is the most dangerous situation and the force values are solved as: $F_{A T}^{\prime}=40 \mathrm{~N}, F_{B X}=450 \mathrm{~N}, F_{B Y}$ $=40 \mathrm{~N}$. Thus, the static simulation will be performed on the model when the cantilever is horizontal.

The column and cantilever models were established in ANSYS with maximum expansion size and analyzed to solve the deformation condition. Figures 6 and 7 show the deformation curves. The simulation results are listed in Table 1.

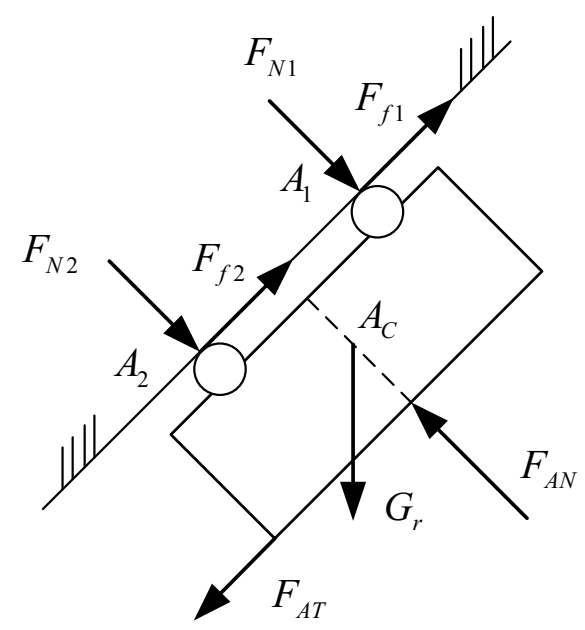

Figure 8 Forces of the wall-climbing robot

According to the results, the maximum stresses are much smaller than the allowable stress so the column and cantilever meet the strength requirement. For further analysis, eigenvalue buckling analysis is performed to confirm whether the column and cantilever are unstable under the working condition. The simulation results show that the critical loads of the first-order eigenvalue buckling of the column and the cantilever are $895.48 \mathrm{~N}$ and $8778.6 \mathrm{~N}$, respectively, which are greater than the working load $(450 \mathrm{~N})$. Therefore, the structure satisfies the stability requirements.

\section{Analysis of Support Performance}

The thrust of the robot is provided by the preload generated when the support mechanism is installed. However, when the robot is in different positions, the force of the mechanism is different, resulting in different deformation of the support mechanism. This deformation will affect the spring compression amount and result in the change in thrust. Therefore, it is necessary to establish a mathematical model to analyze the impact of the deformation of the support mechanism on the thrust.

First, the thrust that maintains a $20 \mathrm{~kg}$ robot without slipping and overturning is calculated. Figure 8 shows the force of the robot when the adsorption force results from the thrust of the support mechanism. The meanings and corresponding values of the symbols are presented in Table 2.

When the robot does not slip, the forces satisfy:

$$
\left\{\begin{array}{l}
F_{A N}=F_{N 1}+F_{N 2}+G_{r} \cos \alpha, \\
F_{A T}+G_{r} \sin \alpha \leq F_{f 1}+F_{f 2}, \\
F_{f 1}=\mu F_{N 1} ; F_{f 2}=\mu F_{N 2} .
\end{array}\right.
$$


Table 2 Parameters of force analysis

\begin{tabular}{lll}
\hline Symbol & Implication & Value \\
\hline$G_{r}$ & Gravity of the robot & $\leq 200 \mathrm{~N}$ \\
$F_{N 1}, F_{N 2}$ & Support force from wall & - \\
$F_{f 1}, F_{f 2}$ & Static friction & - \\
$F_{A T}, F_{A N}$ & Force from cantilever & - \\
$\mu$ & Sliding friction coefficient between rubber and steel & 0.6 \\
$G_{2}$ & Gravity of the cantilever & $\leq 80 \mathrm{~N}$ \\
$L$ & Length between the front and rear wheels of the & $492 \mathrm{~mm}$ \\
& robot & $80 \mathrm{~mm}$ \\
$h$ & Height of the gravity center of the robot & $180 \mathrm{~mm}$ \\
$H$ & Height of the robot & \\
\hline
\end{tabular}

The following equation can be obtained according to Figure 5:

$$
F_{A T}=F_{A T}^{\prime}=G_{2} \sin \alpha \frac{L_{B C}}{L_{A B}} .
$$

Substituting Eq. (4) into Eq. (3) yields:

$$
F_{A N} \geq \frac{1}{\mu}\left(G_{2} \frac{L_{B C}}{L_{A B}}+G_{r}\right) \sin \alpha+G_{r} \cos \alpha .
$$

Therefore, the thrust that maintains the robot without slipping at various positions satisfies the following equation:

$$
\begin{aligned}
& F_{A N} \geq\left(\frac{1}{\mu}\left(G_{2} \frac{L_{B C}}{L_{A B}}+G_{r}\right) \sin \alpha+G_{r} \cos \alpha\right)_{\max } \\
& =\sqrt{\left(\frac{1}{\mu}\left(G_{2} \frac{L_{B C}}{L_{A B}}+G_{r}\right)\right)^{2}+G_{r}^{2}}
\end{aligned}
$$

The result is $447.12 \mathrm{~N}$ after the relevant value substitutions.

When the robot does not rotate around the contact point (A2) between its rear wheel and the wall, the torque about A2 satisfies:

$$
M_{A_{2}}=\frac{L}{2} F_{A N}-L F_{N 1}-\frac{L}{2} G_{r} \cos \alpha-h G_{r} \sin \alpha-H F_{A T} \geq 0,
$$

According to the above analysis, if the support mechanism can provide a supporting force of over $450 \mathrm{~N}$, a robot with a mass of less than $20 \mathrm{~kg}$ will not slide or capsize in any position inside the spherical tank.

Figure 9 illustrates the deformation model of the support mechanism where point $A$ is the connection point between the support mechanism and the robot. $\mathrm{BD}$ represents the initial state of the column and point $B$ is at the center of the tank. $\mathrm{B}^{\prime} \mathrm{D}$ represents the column with deformation and $\mathrm{BB}$ ' describes the deformation

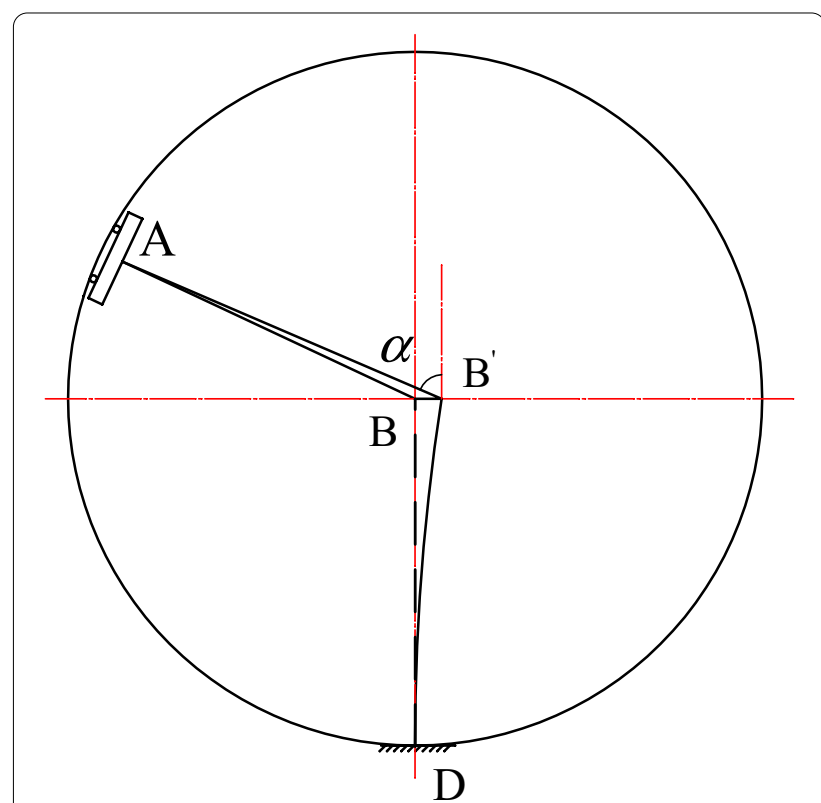

Figure 9 Model with deformation of the mechanism

quantity. BB' is assumed to be horizontal as the deformation mainly occurs in the horizontal direction. Then, each length can be written as:

$$
\begin{aligned}
& l_{-} A B=R-h_{-} r, \\
& l_{A B^{\prime}}=l_{k}-d l_{k}+l_{x}-d l_{x}, \\
& l_{B B^{\prime}}=d l_{s},
\end{aligned}
$$

where $d l_{k}$ is the compressed length of the spring. $d l_{x}$ is the axial deformation of the cantilever under the working pressure. $d l_{s}$ represents the horizontal deformation of the column. These are calculated as follows:

$$
\begin{aligned}
& d l_{k}=\frac{F_{A N}}{k}, \\
& d l_{x}=\frac{F_{A N}}{k_{x}}, \\
& d l_{s}=\frac{F_{B X}}{k_{s}},
\end{aligned}
$$

and, from $\triangle A B B^{\prime}$, the following is satisfied:

$$
l_{A B}^{2}=l_{A B^{\prime}}^{2}+l_{B B^{\prime}}^{2}-2 \times l_{A B^{\prime}} \times l_{B B^{\prime}} \times \cos (90-\alpha) .
$$

The meanings and values of these symbols are listed in Table 3. 
Table 3 Equation parameters

\begin{tabular}{ll}
\hline Parameter & Value \\
\hline Radius of the working spherical tank $R(\mathrm{~mm})$ & 7850 \\
Height of the robot $h_{r}(\mathrm{~mm})$ & 180 \\
Initial length of the spring $I_{k}(\mathrm{~mm})$ & 270 \\
Initial length of the cantilever $I_{x}$ & - \\
Axial stiffness of the cantilever $k_{x}(\mathrm{~N} / \mathrm{mm})$ & 5984.04 \\
Horizontal stiffness of the column $k_{s}(\mathrm{~N} / \mathrm{mm})$ & 18.3 \\
Spring stiffness $k(\mathrm{~N} / \mathrm{mm})$ & 10
\end{tabular}

According to Eq. (2), the horizontal force on the column $F_{B X}$ can be expressed as:

$$
F_{B X}=G_{2} \frac{L_{B C}}{L_{A B}} \sin \alpha \cos \alpha+F_{A N} \sin \alpha .
$$

Substituting the values mentioned above into Eq. (14), the following can be derived:

$$
\begin{aligned}
\left(R-h_{r}\right)^{2}= & \left(l_{k}-\frac{F_{A N}}{k}+l_{x}-\frac{F_{A N}}{k_{x}}\right)^{2} \\
& +\left(\left(G_{2} \frac{L_{B C}}{L_{A B}} \sin \alpha \cos \alpha+F_{A N} \sin \alpha\right) / k_{s}\right)^{2} . \\
& -2 \times\left(l_{k}-\frac{F_{A N}}{k}+l_{x}-\frac{F_{A N}}{k_{x}}\right) \\
& \times\left(\left(G_{2} \frac{L_{B C}}{L_{A B}} \sin \alpha \cos \alpha+F_{A N} \sin \alpha\right) / k_{s}\right) \times \sin \alpha
\end{aligned}
$$

Eq. (16) describes how the thrust provided by the support mechanism varies with angle $\alpha$ as the support mechanism deforms. This value is related to $l_{x}$, the initial length of the cantilever.

The mechanism should be able to pass smoothly through all points of the tank; the initial condition is set such that when the cantilever is vertical the mechanism has no deformation in the horizontal direction; i.e., $d l_{s}=0$ when $\alpha=0^{\circ}$. According to Eq. (16), the following can be deduced:

$$
R-h_{r}=l_{k}-\frac{F_{A N}}{k}+l_{x}-\frac{F_{A N}}{k_{x}} .
$$

Given the initial axial force $F_{A N}=700 \mathrm{~N}$, the initial length of the cantilever is solved as $l_{x}=7534.5 \mathrm{~mm}$.

Substituting the value of $l_{x}$ into Eq. (16), the curve of the thrust $F_{A N}$ with the cantilever angle $2 \alpha$ is solved, as shown in Figure 10. Thus, considering the deformation, the thrust provided by the support mechanism is still over $450 \mathrm{~N}$, which satisfies the design objective. Additionally, Figure 11 shows the curve of the horizontal forces on column $F_{B X}$ with a cantilever angle $2 \alpha$. It can be observed that the maximum horizontal force on the

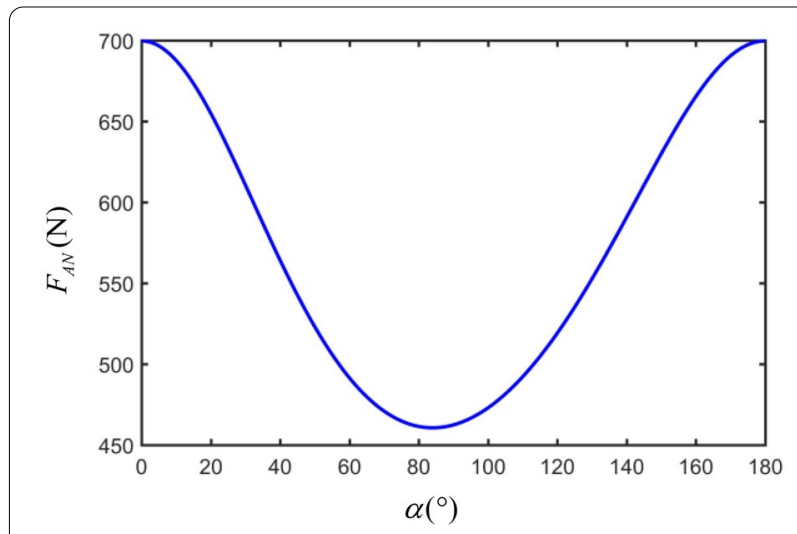

Figure 10 Thrust $F_{A N}$ under mechanism deformation

column is still around $450 \mathrm{~N}$, which is smaller than the critical load $(895.48 \mathrm{~N})$. Hence, the deformation of the column must be within the linear range and no structural instability will occur.

From this analysis, it can be seen that, although the deformation of the support mechanism is inevitable, by adjusting the initial length of the cantilever and changing the compression amount of the spring, the mechanism can still provide sufficient force to ensure stable adsorption for the robot with certain geometric deformation.

\section{Robot Positioning Method}

The support mechanism is a spherical coordinate-type mechanism. The length of the mechanism is fixed and the points reached by the end of the cantilever constitute a spherical surface. Therefore, the positioning problem of the robot is converted into the position solution at the end of the support mechanism. The mathematical model is shown in Figure 12, where Point A represents the position of the robot and lines $\mathrm{AB}$ and $\mathrm{BD}$ represent the cantilever and the column,

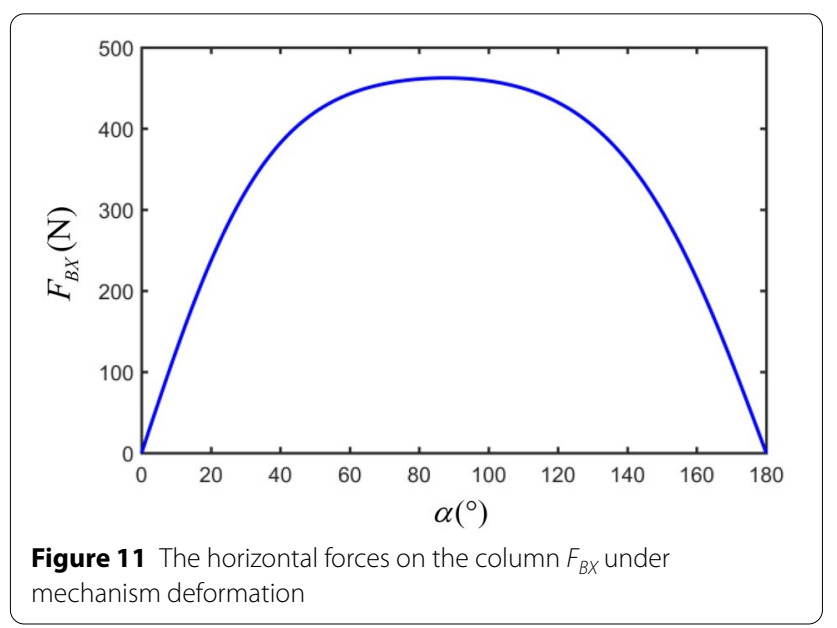




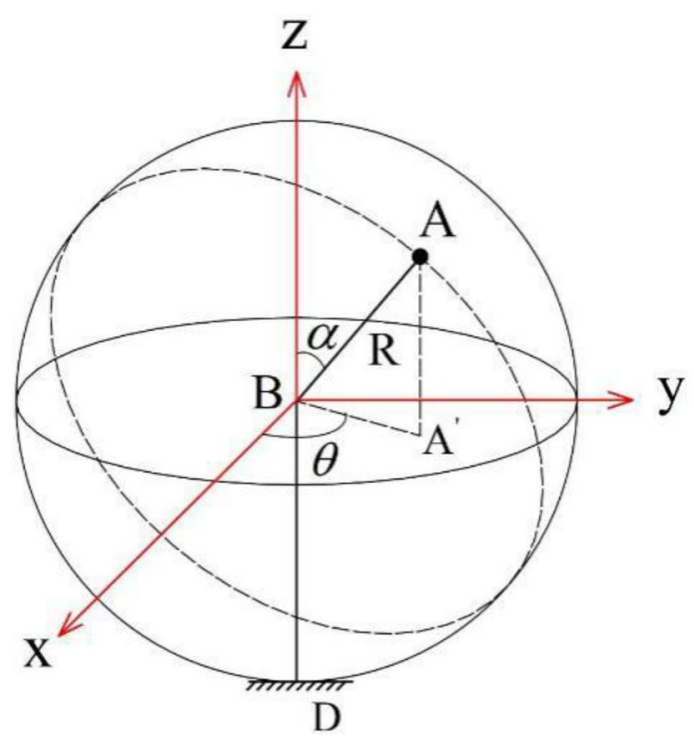

Figure 12 The thrust $F_{\text {AN }}$ under mechanism deformation

respectively. Thus, the three-dimensional position coordinates of the robot can be expressed as follows:

$$
\left\{\begin{array}{l}
x=R \times \sin \alpha \times \cos \theta \\
y=R \times \sin \alpha \times \sin \theta \\
z=R \times \cos \alpha
\end{array}\right.
$$

By detecting the value of $(R, \alpha, \theta)$, the robot at the end of the cantilever can be directly positioned and its absolute coordinate position inside the spherical tank can be calculated.

The positioning method can be realized using two laser range finders and two angular displacement sensors. Angle sensor 1 directly measures the rotation angle of the pitch axis. Owing to size limitations, the rotation range is $-50^{\circ} \sim 90^{\circ}$, corresponding to $\alpha$ from $140^{\circ}$ to $0^{\circ}$. Angle sensor 2 indirectly detects the rotation angle of the rotating axis through a pair of gears with a gear ratio of 50/100 and the rotation ranges from 0 to $360^{\circ}$, corresponding to $\theta$ from 0 to $360^{\circ}$.

Therefore, through the two sets of sensors, the absolute coordinate position of the robot can be obtained and the measurement space satisfies the following constraints:

$$
\left\{\begin{array}{l}
\theta \in\left[0,360^{\circ}\right], \\
\alpha \in\left[0,140^{\circ}\right], \\
R \in[2.3,7.85] .
\end{array}\right.
$$

As shown in Figure 13, the space between the two hemispherical surfaces is the actual measurement space of the support mechanism.

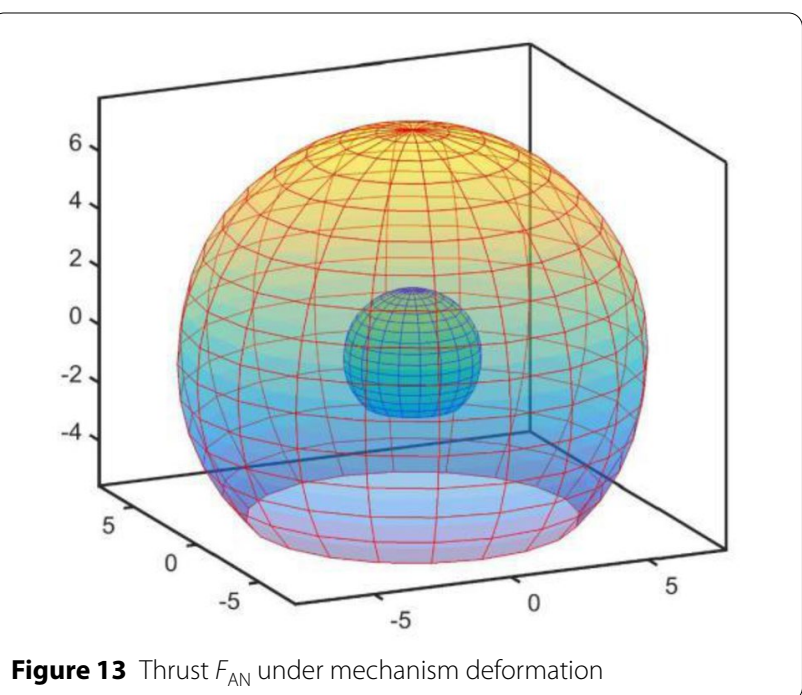

\section{Experiments}

As shown in Figure 14, the prototype of the support mechanism was developed.

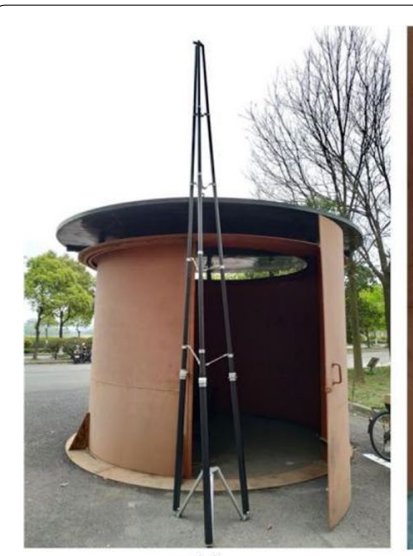

(a)

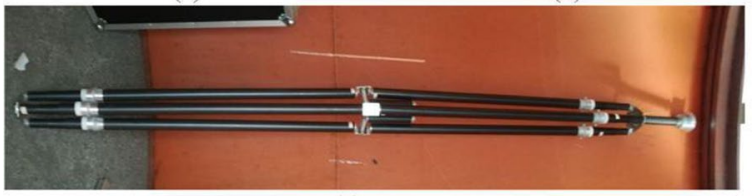

(c)

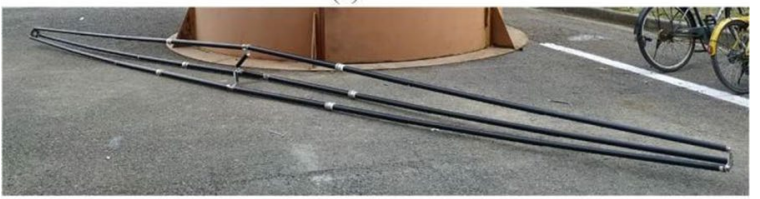

(d)

Figure 14 Prototype of the column and cantilever: a Unfolding column; b Folding column; c Unfolding cantilever; d Folding cantilever 


\subsection{Support Capability Testing}

To detect the pressure exerted by the support mechanism on the wall surface, a pressure sensor is connected at the end of the cantilever, as shown in Figure 15.

By adding the initial compression of the spring and the initial length of the cantilever, different pressures can be detected. The maximum pressure value measured in the experiment was approximately $280 \mathrm{~N}$. To prevent the support mechanism from capsizing or moving, the pressure value will not continue to increase. At this time, there is no obvious deformation of the support mechanism. The experimental results show that the support mechanism can bear a certain supporting force and the supporting force is adjustable within a certain range. The test results demonstrate that the mechanism can satisfy the requirements of stability and safety.

\subsection{Positioning Accuracy Testing}

The support mechanism is designed for a spherical tank; however, when the length of the cantilever is variable, the arbitrary position in the reachable space of the cantilever can be solved. As shown in Figure 16, when the columns of the support mechanism are located on the centerline of the cylinder, the coordinates of any point on cylinder $A(x, y, z)$ can be expressed as:

$$
\left\{\begin{array}{l}
x=l_{A B} \times \sin \alpha \times \cos \theta, \\
y=l_{A B} \times \sin \alpha \times \sin \theta, \\
z=l_{B D}+l_{A B} \times \cos \alpha .
\end{array}\right.
$$

The coordinates can also be determined by its height $h$ and circumference $l$. The relation is satisfied as:

$$
\left\{\begin{array}{l}
h=l_{\mathrm{BD}}+l_{A B} \times \cos \alpha \\
l=R \times\left(\theta-\theta_{0}\right)
\end{array}\right.
$$

where $\theta_{0}$ is the initial rotation angle.

To test the positioning accuracy of the support mechanism, 20 points on the surface of the cylindrical tank were measured by the support mechanism. The result is shown in Figure 17.

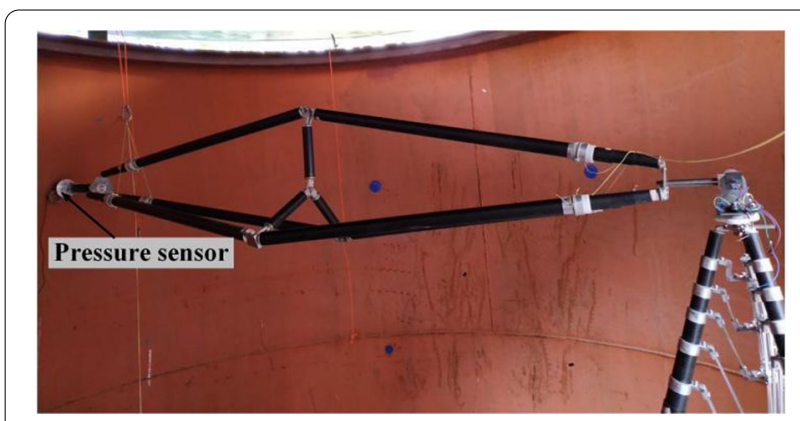

Figure 15 Support capability testing
The average errors of the height and circumference are $3.34 \mathrm{~cm}$ and $1.23 \mathrm{~cm}$, respectively, and the average errors of the two rotation angles are $0.995^{\circ}$ and $0.329^{\circ}$. The two angle sensors are the same; however, because angle sensor 2 transmits from the rotation shaft by gear, the measurement precision of rotation angle 2 is improved. As a result, the height of the points in the system for the cylindrical tank has a much greater error than the perimeter.

\subsection{Comprehensive Testing}

As shown in Figure 18, the support mechanism is connected to a magnetic adsorption robot. The robot moves along the circumference and the support mechanism calculates the trajectory of the robot, as shown in Figure 19. The results indicate that the support mechanism can locate the position of the robot in real-time.

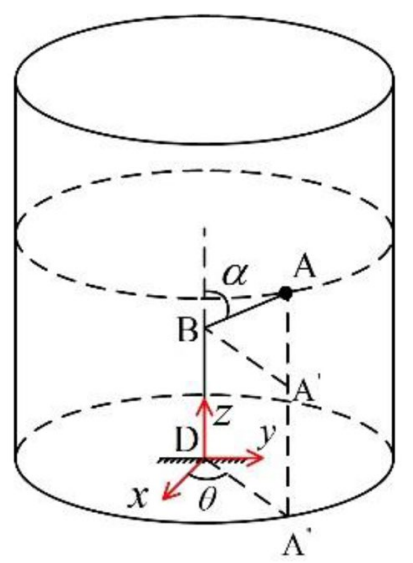

Figure 16 Position calculation model of the cylindrical tank

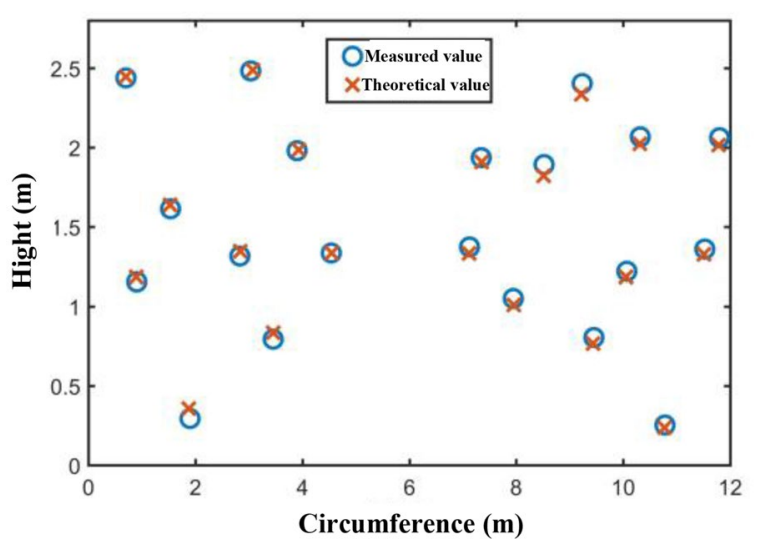

Figure 17 Result of position measurement error 


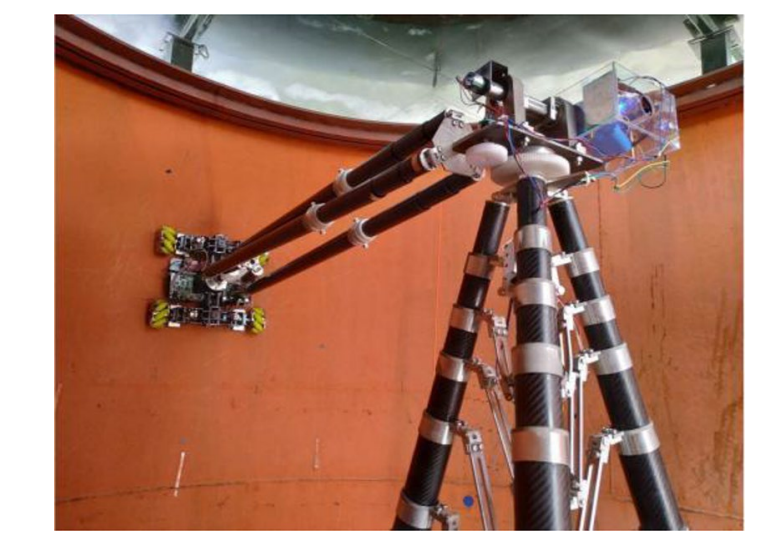

Figure 18 Robot connected with the cantilever

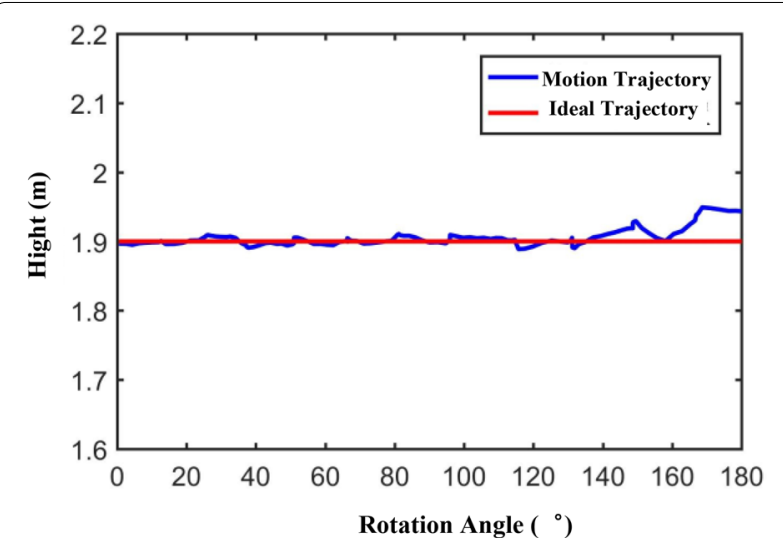

Figure 19 Trajectory of the robot measured by the support mechanism

\section{Conclusions}

(1) In response to the positioning requirements of the spherical tank wall-climbing robot, we proposed a telescopic passive support and positioning mechanism and simulated and analyzed the mechanism deformation.

(2) The force analysis of the supporting and positioning mechanism indicates that the mechanism can provide sufficient force to ensure stable adsorption of the wall-climbing robot.

(3) The mechanism can effectively locate the climbing robot on the inner surface of the spherical tank.

(4) Experiments indicate that the support and positioning mechanism for the wall-climbing robot has suf- ficient support capability and the positioning accuracy can meet the operational requirements.

\section{Acknowledgements}

Not applicable.

\section{Authors' contributions}

CT was in charge of the whole trial; SJ and XW wrote the manuscript; KZ and SS assisted with sampling and laboratory analyses. All authors read and approved the final manuscript.

\section{Authors' Information}

Chunlei Tu, born in 1985, is current an Eng.D candidate at School of Mechanical Engineering, Southeast University, China. He received his master degree on mechanical manufacturing from Zhejiang University, China, in 2011. His research interests include Inspection robotics.

Shanshan Jin, born in 1994, is currently a master candidate at Laboratory of Robotic and BioMechatronics, Southeast University, China.

Kai Zheng, born in 1968, is currently a senior engineer at Special Equipment Safety Supervision Inspection Institute of Jiangsu Province, China. He received his PhD degree from Nanjing University, China, in 2008.

Xingsong Wang, born in 1967, is currently a professor and a PhD candidate supervisor at Laboratory of Robotic and BioMechatronics, Southeast University, China. His main research interests include Biomechatronics, advanced medical equipment, Automation theory and Measurement technology.

Sichong Sun, born in 1991, is currently a PhD candidate at Chongqing University, China, He received his master degree on Mechanical and Energy Engineering from Chongaing University, China, in 2015.

\section{Funding}

Supported by Jiangsu Major Research and Development (Social Development) Project of China (Grant No. BE2016802).

\section{Competing Interests}

The authors declare no competing financial interests.

\section{Author Details}

${ }^{1}$ College of Mechanical Engineering, Southeast University, Nanjing 211189 , China. ${ }^{2}$ Special Equipment Safety Supervision Inspection Institute of Jiangsu Province, Nanjing 210036, China. ${ }^{3}$ College of Power Engineering, Chongqing University, Chongqing 400044, China.

Received: 21 July 2019 Revised: 7 November 2020 Accepted: 23 November 2020

Published online: 05 January 2021

\section{References}

[1] G Lee, H Kim, K Seo, et al. MultiTrack: A multi-linked track robot with suction adhesion for climbing and transition. Robotics and Autonomous Systems, 2015, 72: 207-216.

[2] J Zhu, D Sun, S Tso. Development of a tracked climbing robot. Journal of Intelligent and Robotic Systems, 2002, 35(4): 427-443.

[3] T Felsch, G Strauss, C Perez, et al. Robotized inspection of vertical structures of a solar power plant using NDT techniques. Robotics, 2015, 4(2): 103-119.

[4] C Hillenbrand, D Schmidt, K Berns. CROMSCl: Development of a climbing robot with negative pressure adhesion for inspections. Industrial Robot: An International Journal, 2008, 35(3): 228-237.

[5] D Longo, G Muscato. The Alicia/sup 3/ climbing robot: A three-module robot for automatic wall inspection. Robotics \& Automation Magazine IEEE, $2006,13(1): 42-50$ 
[6] N Sakagami, Y Yumoto, TTakebayashi, et al. Development of dam inspection robot with negative pressure effect plate. Journal of Field Robotics, 2019, 36(8): 1422-1435.

[7] M O Faruq Howlader, T P Sattar. Novel adhesion mechanism and design parameters for concrete wall-climbing robot. IEEE 2015 SAI Intelligent Systems Conference (IntelliSys), London, UK, Nov. 10-11, 2015, https://doi. org/10.1109/intellisys.2015.7361153.

[8] H Leon-Rodriguez, S Hussain, T Sattar. A compact wall-climbing and surface adaptation robot for non-destructive testing. 12th International Conference on Control, Automation and Systems (ICCAS), 2012.

[9] A L C Oliveira, M F Silva, R S Barbosa. Architecture of a wheeled climbing robot with dynamic adjustment of the adhesion system. IEEE 8th International Symposium on Intelligent Systems and Informatics, 2010.

[10] B Li, K Ushiroda, L Yang, et al. Wall-climbing robot for non-destructive evaluation using impact-echo and metric learning SVM. International Journal of Intelligent Robotics and Applications, 2017, 1(3): 255-270.

[11] S Seriani, L Scalera, M Caruso, et al. Upside-down robots: Modeling and experimental validation of magnetic-adhesion mobile systems. Robotics, 2019, 8(2): 41

[12] O Kermorgant. A magnetic climbing robot to perform autonomous welding in the shipbuilding industry. Robotics and Computer-Integrated Manufacturing, 2018, 53: 178-186.

[13] D Schmidt, K Berns. Climbing robots for maintenance and inspections of vertical structures-A survey of design aspects and technologies. Robotics and Autonomous Systems, 2013, 61(12): 1288-1305.

[14] H M La, T H Dinh, N H Pham, et al. Automated robotic monitoring and inspection of steel structures and bridges. Robotica, 2019, 37(5): 947-967.

[15] T Go, T Osawa, T Nakamura. Proposed locomotion strategy for a travelingwave-type omnidirectional wall-climbing robot for spherical surfaces. 2015 IEEE International Conference on Robotics and Biomimetics (ROBIO), 2015.

[16] Z Cai, Z Tao, J Bai, et al. Design of landing platform on climbing robot for a Small Unmanned Aerial Vehicle. IEEE International Conference on Mechatronics \& Automation, 2015.

[17] Q Zhou, X Li. Experimental investigation on climbing robot using rotation-flow adsorption unit. Robotics and Autonomous Systems, 2018, 105: 112-120.

[18] Liu Y, Sun S, Wu X, et al. A wheeled wall-climbing robot with bio-inspired spine mechanisms. Journal of Bionic Engineering, 2015, 12(1): 17-28.

[19] F Xu, F Meng, Q Jiang, et al. Grappling claws for a robot to climb rough wall surfaces: Mechanical design, grasping algorithm, and experiments. Robotics and Autonomous Systems, 2020, 128: 103501

[20] W Fischer, F Tache, R Siegwart. Inspection system for very thin and fragile surfaces, based on a pair of wall climbing robots with magnetic wheels. IEEE/RSJ International Conference on Intelligent Robots and Systems, 2007.
[21] L Kelley, S Ostovari, A B Burmeister, et al. Design and experimental validation of a simple controller for a multi-segment magnetic crawler robot. SPIE, 2015.

[22] A San-Millan. Design of a teleoperated wall climbing robot for oil tank inspection. 23rd Mediterranean Conference on Control and Automation, 2015, https://doi.org/10.1109/med.2015.7158759.

[23] R Wang, Y Kawamura. An automated sensing system for steel bridge inspection using GMR sensor array and magnetic wheels of climbing robot. Journal of Sensors, 2016, 2016: 1-15.

[24] W Shen, J Gu, Y Shen. Proposed wall climbing robot with permanent magnetic tracks for inspecting oil tanks. IEEE International Conference on Mechatronics and Automation, 2005.

[25] K Nagaya, T Yoshino, M Katayama, et al. Wireless piping inspection vehicle using magnetic adsorption force. IEEE/ASME Transactions on Mechatronics, 2012, 17(3): 472-479.

[26] F Fang, TWang, B Li. Analysis and design of electromagnetic vehicles climbing on steel plates. IEEE International Conference on Mechatronics and Automation (ICMA), 2015.

[27] H Huang, D Li, Z Xue, et al. Design and performance analysis of a tracked wall-climbing robot for ship inspection in shipbuilding. Ocean Engineering, 2017, 131: 224-230

[28] M Tavakoli, P Lopes, L Sgrigna, et al. Motion control of an omnidirectional climbing robot based on dead reckoning method. Mechatronics, 2015, 30: 94-106.

[29] M Tavakoli, J Lourenço, C Viegas, et al. The hybrid OmniClimber robot: Wheel based climbing, arm based plane transition, and switchable magnet adhesion. Mechatronics, 2016, 36: 136-146.

[30] M Tavakoli, C Viegas, L Marques, et al. OmniClimbers: Omni-directional magnetic wheeled climbing robots for inspection of ferromagnetic structures. Robotics and Autonomous Systems, 2013, 61(9): 997-1007.

[31] M Tavakoli, C Viegas. Analysis and application of dual-row omnidirectional wheels for climbing robots. Mechatronics, 2014, 24(5): 436-448.

[32] J Li, X S Wang. Novel omnidirectional climbing robot with adjustable magnetic adsorption mechanism. 2016 23rd International Conference on Mechatronics and Machine Vision in Practice (M2VIP), 2016.

[33] K Zheng, J Li, C Tu, et al. Two opposite sides synchronous tracking X-ray based robotic system for welding inspection. 2016 23rd International Conference on Mechatronics and Machine Vision in Practice (M2VIP), 2016.

[34] C Tu, X Li, J Li, et al. Bilateral laser vision tracking synchronous inspection robotic system. 2017 Far East NDT New Technology \& Application Forum (FENDT), 2017.

[35] G Liang, K Zheng, C Tu, et al. Existing weld seam recognition based on image processing. Far East NDT New Technology and Application Forum, 2017

\section{Submit your manuscript to a SpringerOpen ${ }^{\odot}$ journal and benefit from:}

- Convenient online submission

- Rigorous peer review

- Open access: articles freely available online

- High visibility within the field

Retaining the copyright to your article

Submit your next manuscript at springeropen.com 